Western Washington University Western CEDAR

2004

\title{
A Counterexample in Sturm-Liouville Completeness Theory
}

\author{
Paul Binding \\ University of Calgary \\ Branko Ćurgus \\ Western Washington University, branko.curgus@wwu.edu
}

Follow this and additional works at: https://cedar.wwu.edu/math_facpubs

Cart of the Mathematics Commons

\section{Recommended Citation}

Binding, Paul and Ćurgus, Branko, "A Counterexample in Sturm-Liouville Completeness Theory" (2004). Mathematics. 9.

https://cedar.wwu.edu/math_facpubs/9

This Article is brought to you for free and open access by the College of Science and Engineering at Western CEDAR. It has been accepted for inclusion in Mathematics by an authorized administrator of Western CEDAR. For more information, please contact westerncedar@wwu.edu. 


\title{
A counterexample in Sturm-Liouville completeness theory
}

\author{
Paul Binding \\ Department of Mathematics and Statistics, University of Calgary, \\ Calgary, Alberta T2N 1N4, Canada (binding@ucalgary.ca)

\section{Branko Ćurgus} \\ Department of Mathematics, Western Washington University, \\ Bellingham, WA 98226, USA (curgus@cc.wwu.edu)
}

(MS received 2 October 2002; accepted 10 September 2003)

\begin{abstract}
We give an example of an indefinite weight Sturm-Liouville problem whose eigenfunctions form a Riesz basis under Dirichlet boundary conditions but not under anti-periodic boundary conditions.
\end{abstract}

\section{Introduction}

Completeness of eigenfunctions for Sturm-Liouville equations of the form

$$
\ell(y):=-\left(p y^{\prime}\right)^{\prime}+q y=\lambda r y
$$

on $[-1,1]$ was already investigated for the case of positive weight functions $r$ in the 1830s. In a modern setting, one seeks complete orthonormal bases in the weighted Hilbert space $L_{2, r}(-1,1)$ with inner product given by

$$
(y, z)_{r}=\int_{-1}^{1} r y \bar{z} .
$$

For the purposes of this note, it will suffice to consider regular positive-definite $\ell$ with self-adjoint boundary conditions and $r \in L_{\infty}(-1,1)$.

Problems with indefinite $r$ were studied around the turn of the 20th century, and orthonormal bases of eigenfunctions were examined in a Hilbert space with the Dirichlet inner product generated by $\ell$. We remark that early work on all the above problems usually assumed Dirichlet boundary conditions $y(1)=y(-1)=0$ for $(1.1)$, but the theory now encompasses arbitrary self-adjoint boundary conditions.

Around 1970, 'half-range' completeness for certain forward-backward equations led to renewed interest in the $L_{2, r}(-1,1)$ setting, but now for equations (1.1) with indefinite $r$. An important step in the corresponding analysis of completeness is the production of a Riesz basis of eigenfunctions. In this case, $L_{2, r}(-1,1)$ is a Krein space, but the Riesz basis is taken with respect to the underlying Hilbert space inner product (with $r$ replaced by $|r|$ in (1.2)).

A standard reference in this area is [2], where Beals produces a basis (which turns out to be a Riesz basis) of eigenfunctions for a certain class of weight functions $r$. In 
particular, certain 'extra' conditions on $r$ are imposed at the single turning point, i.e. where $r$ changes sign. As Beals points out, the analysis extends to finitely many turning points, but there is an example of Pyatkov [9] with infinitely many turning points and no Riesz basis of eigenfunctions. Also, Volkmer [11] has shown that some 'extra' condition is necessary for the Beals result to hold. Explicit counterexamples, based on Volkmer's ideas, can be found in [1] and [6]. We remark that all these works assume Dirichlet boundary conditions at 1 and -1 .

We are aware of two works on completeness in $L_{2, r}(-1,1)$ for problems of the form (1.1) with indefinite $r$ and general self-adjoint boundary conditions. One is [5], which involves 'extra' conditions on $r$ at the turning points and at the boundary. The other [10] assumes 'extra' conditions only at the turning point. We shall show by example that some 'extra' condition is indeed required in general at the boundary, so [10, theorems 3.1, 3.2] are incorrect as stated. An intuitive explanation for our example can be given as follows. There is a turning point at $x=0$, where $r$ satisfies an 'extra' condition, and the Dirichlet problem has a Riesz basis of eigenfunctions. When the boundary conditions are not separated, however, the points 1 and -1 are linked and (1.1) can be thought of as living on a circle, with a second turning point at \pm 1 . In our example, there is no 'extra' condition on $r$ at \pm 1 and there is no Riesz basis of eigenfunctions for the anti-periodic problem.

We shall adapt Volkmer's strategy, which is to establish an inequality, to be denoted by $I$ (see (2.3)), satisfied by a class $\mathcal{V}$ (see (2.4)) of absolutely continuous functions. While such inequalities have been examined by various authors (and they are sometimes called HELP inequalities, cf. [3]), Volkmer emphasizes the role of the boundary conditions on the functions in $\mathcal{V}$. Indeed, he shows that $I$ is false in the absence of boundary conditions, true independently of (1.1) if two Neumann-type conditions are imposed, and true if one such condition is imposed and there is a Riesz basis of Dirichlet eigenfunctions.

In $\S 2$, we shall show that $I$ also holds if there is a Riesz basis of anti-periodic eigenfunctions and another boundary condition is imposed (in addition to Volkmer's, giving a smaller class $\mathcal{V}_{0}$ ). We then show that an example of (1.1) given in [1] generates functions in $\mathcal{V}_{0}$ and failing $I$, so it does not have a Riesz basis of anti-periodic eigenfunctions. In $\S 3$, we modify this example so as to have the properties in the abstract. This shows, incidentally, that $I$, even on the larger class $\mathcal{V}$, is not sufficient for a Riesz basis of anti-periodic eigenfunctions.

Finally, we note that mixed boundary conditions raise the possibility of double eigenvalues. If there are infinitely many such eigenvalues, then there could be some sets of (normalized) eigenfunctions that form Riesz bases and other sets that do not. In $\S 4$, we prove that this difficulty cannot occur. In fact, all the eigenproblems considered here have only simple eigenvalues.

\section{A necessary condition for a Riesz basis}

Throughout, $w$ will denote an odd function in $L_{\infty}(-1,1)$. Here, odd will mean that $w(-x)=-w(x)$ for all $x \neq 0$. We use $\|\cdot\|$ to denote the norm in $L_{2}(-1,1)$.

We define an operator $A$ in $L_{2}(-1,1)$ on

$$
\operatorname{dom}(A)=\left\{f \in W_{2}^{2}(-1,1): f(1)+f(-1)=0, f^{\prime}(1)+f^{\prime}(-1)=0\right\}
$$


by

$$
A f=-f^{\prime \prime} .
$$

It is well known (see [12]) that $A$ is self-adjoint with compact resolvent, and an easy calculation shows that the eigenvalues are all positive.

We shall consider the weighted eigenvalue problem

$$
A f=\lambda w f,
$$

which can be studied in a natural way as the standard eigenvalue problem for the positive self-adjoint operator $w^{-1} A$ in the weighted space $L_{2, w}(-1,1)$.

The first step in our analysis is the following modification of $[11, \S 4]$. We use the notation

$$
K(h)=\frac{\left(\int_{0}^{1}\left|h^{\prime}\right|^{2} / w\right)^{2}}{\int_{0}^{1}|h|^{2} \int_{0}^{1}\left|\left(h^{\prime} / w\right)^{\prime}\right|^{2}}
$$

whenever the right-hand side makes sense.

LEMMA 2.1. If $w$ is an odd essentially bounded function on $[-1,1]$ such that $x w(x)>0$ for all non-zero $x$ and if there is a Riesz basis of eigenfunctions in $L_{2, w}(-1,1)$ for the eigenvalue problem (2.1), then there exists $c>0$ such that

$$
K(h)<c^{2}
$$

for all non-zero $h$ such that

$$
h \in A C(0,1), \quad \frac{h^{\prime}}{w} \in W_{2}^{1}(0,1), \quad\left(\frac{h^{\prime}}{w}\right)(1)=0
$$

and

$$
h(1)=0 .
$$

Proof. Since $A$ has a positive compact inverse in $L_{2}(-1,1)$, theorem 2.2 of [1]] applies and gives $c$ such that

$$
\int_{-1}^{1}\left|w\left\|\left.f\right|^{2} \leqslant c\right\| A^{1 / 2} g\|\| A^{1 / 2} f \|\right.
$$

for all $f \in \operatorname{dom}\left(A^{1 / 2}\right)$ and $g=A^{-1}(w f)$.

Let $h$ satisfy $(2.4)$ and (2.5). Extend $h$ to an even function on $[-1,1]$ and put $f:=-h^{\prime} / w$. By $(2.4)$, we have $f(-1)=f(1)=0$ and therefore $f$ belongs to

$$
\operatorname{dom}\left(A^{1 / 2}\right)=\left\{y \in W_{2}^{1}(-1,1): y(-1)+y(1)=0\right\}
$$

$($ see $[7, \S 7])$. Moreover,

$$
\left\|A^{1 / 2} f\right\|^{2}=\int_{-1}^{1}\left|f^{\prime}\right|^{2} .
$$

Solving $-g^{\prime \prime}=w f=-h^{\prime}$ for $g \in \operatorname{dom}(A)$, we obtain

$$
g(x)=\int_{0}^{x} h(t) \mathrm{d} t, \quad x \in[-1,1] .
$$


Since $g \in \operatorname{dom}(A)$ and $h(-1)=h(1)=0$, integration by parts yields

$$
\left\|A^{1 / 2} g\right\|^{2}=\int_{-1}^{1} A g \bar{g}=\int_{-1}^{1} w f \bar{g}=-\int_{-1}^{1} h^{\prime} \bar{g}=\int_{-1}^{1}|h|^{2} .
$$

Substituting (2.7) and (2.9) in (2.6), we obtain

$$
\left(\int_{-1}^{1}|w||f|^{2}\right)^{2} \leqslant c^{2} \int_{-1}^{1}|h|^{2} \int_{-1}^{1}\left|f^{\prime}\right|^{2},
$$

and since all the integrands are even functions, it follows that $K(h) \leqslant c^{2}$ for all $h$ satisfying (2.4) and (2.5).

The next step is to show that functions $w$ exist that fail the conclusion of lemma 2.1. An analogous approach has already been used for Dirichlet boundary conditions in [1] and [6] via Volkmer's version of lemma 2.1 (see [11, §4]), which includes conditions (2.4), but not (2.5).

THEOREM 2.2. There is an odd essentially bounded function $w$ defined on $[-1,1]$ with $x w(x)>0$ for all non-zero $x$, continuously differentiable near 1 , and such that no set of eigenfunctions of the problem $A f=\lambda w f$ is a Riesz basis of $L_{2, w}(-1,1)$.

Proof. In [1, theorem 1], a condition (see [1, eqn (5)]) for $w$ is given under which lemma 2.1 fails for the set of functions $h$ that satisfy (2.4) (but not (2.5)). The proof of [1, theorem 1] involves functions $f_{n}$ defined by

$$
f_{n}(x)=-\int_{x}^{1} g_{n}, \quad x \in[0,1],
$$

and these evidently vanish at $x=1$. The functions $f_{n}$ play the role of our $h$, and so our additional condition (2.5) is automatically incorporated in the construction in [1, theorem 1]. Thus [1, theorem 1] gives a sufficient condition for $w$ under which no set of eigenfunctions of the problem $A f=\lambda w f$ is a Riesz basis of $L_{2, w}(-1,1)$. Therefore, example 2 of [1] applies to our situation.

REMARKS 2.3. It follows from the construction in [1] that the function $w$ in theorem 2.2 can, in fact, be selected to be an arbitrary (essentially bounded) function on any interval $[\delta, 1], 0<\delta<1$. In view of this, the set of functions $w$ as above is dense in $L_{2, w}$ (and, indeed, in other spaces (see [1, theorem 2] for the Dirichlet case)). In $[11, \S 5]$, Volkmer gave a similar Dirichlet density result using (2.4) alone.

\section{Boundary conditions and Riesz bases}

In this section we modify the construction in $\S 2$ to produce the example promised in the abstract.

Let $w$ be the essentially bounded function whose existence is established in theorem 2.2. Define $S$ on $L_{2, w}(-1,1)$ by

$$
(S f)(x)=f(x-\operatorname{sgn} x), \quad x \in[-1,1],
$$


where, for definiteness, we take $\operatorname{sgn} 0=1$. Then $r:=S w$ is essentially bounded and odd. Since $S^{2} f=f$ for all $f \in L_{2, w}(-1,1)$, we have $S r=w$. We also define $Q$ on $L_{2, w}(-1,1)$ by

$$
(Q f)(x)=(S f)(x) \operatorname{sgn} x .
$$

The operator $Q$ will be our main tool below and it enjoys the following properties.

\section{LEMMA 3.1.}

(a) Operator $Q$ is a unitary isomorphism between the Hilbert spaces $L_{2,|w|}(-1,1)$ and $L_{2,|r|}(-1,1)$.

(b) If $f \in \operatorname{dom}(A)$, then $Q f \in \operatorname{dom}(A)$ and $(Q f)^{\prime \prime}=Q\left(f^{\prime \prime}\right)$ a.e.

(c) If $f \in \operatorname{dom}(A)$, then $Q(r f)=w Q f$ a.e.

Proof. (a) If $f, g \in L_{2,|w|}(-1,1)$, we calculate

$$
\begin{aligned}
\int_{-1}^{1}|r| Q f \bar{Q} g & =\int_{-1}^{1} S(|w| f \bar{g}) \\
& =\int_{0}^{1}|w| f \bar{g}+\int_{-1}^{0}|w| f \bar{g} \\
& =\int_{-1}^{1}|w| f \bar{g} .
\end{aligned}
$$

Thus $Q$ is an isometry. Now suppose that $g \in L_{2,|r|}(-1,1)$ and redefine $g$ on the null set $\{-1,0,1\}$ by $g(-1)=g(0)=g(1)=0$. If we define $f=Q g$, then it is easily checked that $g=Q f$ and $f \in L_{2,|w|}(-1,1)$, so $Q$ maps $L_{2,|w|}(-1,1)$ onto $L_{2,|r|}(-1,1)$ and hence is unitary.

(b) The continuity of $Q f$ and $(Q f)^{\prime}$ at zero follow from the boundary conditions on $f \in \operatorname{dom}(A)$, and conversely (interchanging the roles of $f$ and $Q f$ ). The remaining contentions are straightforward.

(c) Since $S r=S^{2} w=w$, the result follows from

$$
Q(r f)(x)=S(r f)(x) \operatorname{sgn} x=(S r)(x)(S f)(x) \operatorname{sgn} x=w(x)(Q f)(x) .
$$

Now we are ready for the main result.

THEOREM 3.2. There exists odd essentially bounded $r$, with $x r(x)>0$ for all nonzero $x \in(-1,1)$, continuously differentiable near 0 and such that

(a) appropriately normalized eigenfunctions of the Dirichlet problem

$$
-f^{\prime \prime}=\lambda r f
$$

with $f(-1)=f(1)=0$ form a Riesz basis of $L_{2,|r|}(-1,1)$; but

(b) no set of eigenfunctions of the corresponding anti-periodic problem

$$
A f=\lambda r f
$$

forms such a basis. 
Proof. (a) Since the function $w$ is continuously differentiable in the interval $[\delta, 1]$, $0<\delta<1$, it follows that the function $r$ is continuously differentiable on $[-1+\delta, 0) \cup(0,1-\delta]$. Thus $[5$, theorem 3.6] shows that the normalized eigenfunctions of the Dirichlet problem form a Riesz basis in $L_{2,|r|}(-1,1)$.

(b) Suppose there is a Riesz basis of eigenfunctions $f_{n}$ for $(3.2)$ in $L_{2,|r|}(-1,1)$. By lemma 3.1 (a), the functions $Q f_{n}$ form a Riesz basis of $L_{2,|w|}(-1,1)$ and, by parts (b) and (c) of lemma 3.1, they satisfy

$$
A Q f_{n}=\lambda w Q f_{n} .
$$

This evidently contradicts theorem 2.2 , and the proof is complete.

REMARKs 3.3. As in $\S 2$, various modifications are possible. By virtue of [5, theorem 3.6], Dirichlet conditions can be replaced by any separated boundary conditions. One can also restate the results in the Naimark framework [8] as follows. Denote by $W$ the operator in $L_{2, w}(-1,1)$ defined on

$$
\begin{aligned}
& \operatorname{dom}(W)=\left\{f \in L_{2, w}(-1,1): f, f^{\prime} \in A C[-1,1],(1 / w) f^{\prime \prime} \in L_{2, w}(-1,1)\right. \\
& f(1)+f(-1)\left.=0, f^{\prime}(1)+f^{\prime}(-1)=0\right\}
\end{aligned}
$$

by $W f=-(1 / w) f^{\prime \prime}$, and denote by $R$ the analogous operator with $w$ replaced by $r$. Then $Q W=R Q$, so $W$ and $R$ are unitarily similar and therefore if either operator has a Riesz basis of eigenfunctions, so must the other.

\section{Simplicity of the eigenvalues}

As mentioned in $\S 1$, if infinitely many double eigenvalues $\lambda_{n}$ exist, then the question of whether the (normalized) eigenfunctions form a Riesz basis can be ambiguous. Specifically, if the angles $\alpha_{n}$ in $L_{2,|r|}(-1,1)$ between the two specified eigenfunctions at $\lambda_{n}$ tend to 0 as $\lambda_{n} \rightarrow \infty$, then the eigenfunctions do not form a Riesz basis, even though other choices (for which $\alpha_{n}$ do not tend to 0) could form a Riesz basis. We mention the related (abstract) example in [4], where angles between pairs of eigenvectors must tend to 0, preventing existence of a Riesz basis.

Below we prove that all the eigenvalues of the problems considered earlier are, in fact, simple.

LEMma 4.1. Let $v:[0,1] \rightarrow \mathbb{R}$ be a positive essentially bounded measurable function. Then the unique solutions of the initial-value problems

$$
\begin{aligned}
& -y^{\prime \prime}=v y, \quad y(0)=0, \quad y^{\prime}(0)=1, \\
& z^{\prime \prime}=v z, \quad z(0)=0, \quad z^{\prime}(0)=1
\end{aligned}
$$

satisfy $z(t)>y(t)$ for all $t \in(0,1]$.

Proof. We write

$$
s=z+y \quad \text { and } \quad d=z-y
$$

for the sum and difference of $z$ and $y$. We note that

$$
s(0)=d(0)=d^{\prime}(0)=0 \quad \text { and } \quad s^{\prime}(0)=2 .
$$

Thus $s(t)>0$ for sufficiently small $t>0$. 
Arguing by contradiction, we assume that there is $b \leqslant 1$ such that $s(t)>0$ for $0<t<b$, but $s(b)=0$. Then

$$
d^{\prime}(t)=\int_{0}^{t} v s>0
$$

So

$$
d(t)=\int_{0}^{t} d^{\prime}>0
$$

for all $t \in(0, b]$. Moreover,

$$
s^{\prime}(t)=2+\int_{0}^{t} v d>0
$$

So

$$
s(b)=\int_{0}^{b} s^{\prime}>0 .
$$

This is the desired contradiction. Thus $s>0$, and then, from (4.3), $d>0$ also, on $(0,1]$.

Proposition 4.2. Let $r$ be an odd essentially bounded measurable function defined on $[-1,1]$ such that $x r(x)>0$ for all non-zero $x \in[-1,1]$. Then the eigenvalues of the problems (3.1) and (3.2) in theorem 3.2 are real, symmetric with respect to 0 and simple.

Proof. It is standard that the eigenvalues of the Dirichlet problem (3.1) are real and simple. The fact that all the eigenvalues of (3.2) are real follows from [5, propositions 1.1, 2.3 and 2.6]. To see that the eigenvalues of both problems are symmetric with respect to 0 , replace $f(t)$ by $f(-t), t \in[-1,1]$, and $\lambda$ by $-\lambda$.

It remains to prove the simplicity of the eigenvalues of (3.2). By the symmetry expressed above, it is sufficient to consider the positive eigenvalues, so let $\lambda$ be a positive double eigenvalue of (3.2). Then the space of all $f$ such that

$$
\begin{aligned}
-f^{\prime \prime} & =\lambda r f, \\
f(1)+f(-1) & =0, \\
f^{\prime}(1)+f^{\prime}(-1) & =0,
\end{aligned}
$$

is two dimensional. Equivalently, all solutions of (4.4) satisfy (4.5) and (4.6).

Let $f=z$ be the unique solution of (4.4) such that $z(0)=0$ and $z^{\prime}(0)=1$, and define $y(x):=-z(-x), x \in[0,1]$. Then, with $v=\lambda r, z$ is a solution of (4.1) and $y$ is a solution of (4.2). By lemma 4.1, it follows that $z(1)>-z(-1)$, that is, $z(1)+z(-1)>0$. Thus $f=z$ satisfies (4.4), but not (4.5), and we have a contradiction.

REMARK 4.3. With the aid of different initial conditions in (4.1) and (4.2) of lemma 4.1, similar reasoning shows that the eigenvalues of the periodic problem

$$
-f^{\prime \prime}=\lambda r f, \quad f(1)-f(-1)=0, \quad f^{\prime}(1)-f^{\prime}(-1)=0
$$

are also simple. In particular, all the eigenvalues of (3.2) and (4.7) are simple for the function $r(x)=\operatorname{sgn}(x), x \in[-1,1]$. It is interesting to observe that all the 
non-zero eigenvalues of the corresponding definite problems (with $|r|=1$ instead of $r$ ), namely,

$$
\begin{aligned}
& -f^{\prime \prime}=\lambda f, \quad f(1)+f(-1)=0, \quad f^{\prime}(1)+f^{\prime}(-1)=0, \\
& -f^{\prime \prime}=\lambda f, \quad f(1)-f(-1)=0, \quad f^{\prime}(1)-f^{\prime}(-1)=0,
\end{aligned}
$$

are double.

\section{Acknowledgments}

Research supported by I. W. Killam Foundation and NSERC of Canada.

\section{References}

1 N. L. Abasheeva and S. G. Pyatkov. Counterexamples in indefinite Sturm-Liouville problems. Siberian Adv. Math. 7 (1997), 1-8.

2 R. Beals. Indefinite Sturm-Liouville problems and half-range completeness. J. Diff. Eqns 56 (1985), 391-407.

3 C. Bennewitz. A general version of the Hardy-Littlewood-Pólya-Everitt (HELP) inequality. Proc. R. Soc. Edinb. A 97 (1984), 9-20.

4 P. Binding and R. Hryniv. Full- and partial-range completeness. Operator Theory Adv. Appl. 130 (2001), 121-133.

5 B. Ćurgus and H. Langer. A Krein space approach to symmetric ordinary differential operators with an indefinite weight function. J. Diff. Eqns 79 (1989), 31-61.

6 A. Fleige. A counterexample to completeness properties for indefinite Sturm-Liouville problems. Math. Nachr. 190 (1998), 123-128.

7 M. G. Krein. Theory of self-adjoint extensions of semibounded Hermitian operators and its applications. II. Mat. Sb. 21 (1947), 365-404.

8 M. A. Naimark. Linear differential operators. Part II. Linear differential operators in Hilbert space (New York: Frederick Ungar, 1968).

9 S. G. Pyatkov. Some properties of eigenfunctions of linear pencils. Math. Notes 51 (1992), 90-95.

10 S. G. Pyatkov. Interpolation of some function spaces and indefinite Sturm-Liouville problems. Operator Theory Adv. Appl. 102 (1998), 179-200.

11 H. Volkmer. Sturm-Liouville problems with indefinite weights and Everitt's inequality. Proc. R. Soc. Edinb. A 126 (1996), 1097-1112.

12 J. Weidmann. Spectral theory of ondinary differential operators. Lecture Notes in Mathematics, vol. 1258 (Springer, 1987).

(Issued 7 May 2004) 